\title{
Efficient Optimization of Gluconobacter oxydans Based on Protein Scaffold-Trimeric CutA to Enhance the Chemical Structure Stability of Enzymes for the Direct Production of 2-Keto- L-gulonic Acid
}

\author{
Lili Gao $\mathbb{D}^{1},{ }^{1}$ Yuefeng Liu, ${ }^{2}$ Xiaoyu Zhang, ${ }^{1}$ and Hongsheng Zhang ${ }^{1}$ \\ ${ }^{1}$ Hebei Key Laboratory of Applied Chemistry, \\ Heavy Metal Deep-Remediation in Water and Resource Reuse Key Lab of Hebei Province, \\ School of Environmental and Chemical Engineering, Yanshan University, Qinhuangdao 066004, China \\ ${ }^{2}$ Qinhuangdao Vocational and Technical College, Qinhuangdao 066100, China
}

Correspondence should be addressed to Lili Gao; gllqhd@163.com

Received 7 June 2020; Accepted 10 July 2020; Published 4 August 2020

Academic Editor: Peizhi Guo

Copyright ( $\odot 2020$ Lili Gao et al. This is an open access article distributed under the Creative Commons Attribution License, which permits unrestricted use, distribution, and reproduction in any medium, provided the original work is properly cited.

\begin{abstract}
2-Keto-L-gulonic acid (2-KLG), the direct precursor of vitamin C, is produced by a two-step fermentation route from D-sorbitol in industry. However, this route is a complicated mix-culture system which involves three bacteria. Thus, replacement of the conventional two-step fermentation process with a one-step process could be revolutionary in vitamin C industry. The one-step fermentation of 2-keto-L-gulonic acid (2-KLG) has been achieved in our previous study; $32.4 \mathrm{~g} / \mathrm{L}$ of 2-KLG production was obtained by the one-step strain G. oxydans/pGUC-tufB-sdh-GGGGS-sndh after $168 \mathrm{~h}$. In this study, L-sorbose dehydrogenase $(\mathrm{SDH})$ and L-sorbosone dehydrogenase (SNDH) were expressed in G. oxydans after the codon optimization. Furthermore, the trimeric protein CutA was used to improve the chemical structure stability of SDH and SNDH. The recombinant strain $G$. oxydans/pGUC-tufB-SH3-sdh-GGGGS-sndh-tufB-SH3 lig $_{\text {- }}$ (GGGGS) ${ }_{2}$-cutA produced $40.3 \mathrm{~g} / \mathrm{L}$ of 2-KLG after $168 \mathrm{~h}$. In addition, the expression levels of the cofactor PQQ were enhanced to further improve 2-KLG production. With the stepwise metabolic engineering of G. oxydans, the final 2-KLG production was improved to $42.6 \mathrm{~g} / \mathrm{L}$. The efficient one-step production of 2-KLG was achieved, and the final one-step industrial-scale production of 2-KLG is drawing near.
\end{abstract}

\section{Introduction}

Vitamin C (L-ascorbic acid, L-AA) is widely used in pharmaceutical, food, beverages, cosmetics, and feed industries [1]. The most successful route for industrial production of vitamin $\mathrm{C}$ is the classical two-step fermentation process. The fermentation process contains one-step conversion from $\mathrm{D}$-sorbitol to $\mathrm{L}$-sorbose by Gluconobacter oxydans, followed by another step of converting L-sorbose to 2-keto-gulonic acid (2-KLG), the precursor of vitamin C, by a mixed culture system of Ketogulonicigenium vulgare and Bacillus megaterium. K. vulgare is difficult to culture alone and it possesses a rather low production capacity of 2-KLG. The accompany bacterium $B$. megaterium does not produce
2-KLG, but it can promote the growth and 2-KLG production of $K$. vulgare [2]. Over the years, researchers have attempted to improve fermentation processes in many ways; tremendous advances have been achieved in microbial production of vitamin C [2-5]. However, the culture broth from the "first step" by G. oxydans containing L-sorbose needs to be transferred to another bioreactor, added with other culture media, and sterilized for the second time. The addition process involves three microorganisms which significantly increases the cost of both raw materials and energy requirement [6]. Besides, the mix-culture system makes the process optimization difficult.

In consideration of problems presented above in the two-step fermentation process, using D-glucose or 
D-sorbitol as a carbon source for the production of 2-KLG in one-step fermentation process would be more cost-effective and it will be a revolutionary advancement in the vitamin $\mathrm{C}$ industry. Although the strain which can catalyze D-glucose or D-sorbitol to 2-KLG efficiently by single strain fermentation has not been found, advances in biochemistry and recombinant DNA technology, together with the genomic revolution, have promoted the construction of the direct microbial processes to $2-\mathrm{KLG}$ via genetic engineering $[1,7]$. In the earlier study, membrane-bound sorbose dehydrogenase and cytosolic sorbosone dehydrogenase were cloned from G. oxydans T-100 and expressed in G. oxydans G624, which is able to accumulate L-sorbose. The recombinant strain produced $88 \mathrm{~g} / \mathrm{L}$ of 2 -KLG from D-sorbitol [8]. However, there was no research about one-step fermentation over the last decade, and the results obtained in our previous study showed that the expression of SDH and SNDH genes in G. oxydans could only result in a yield of 2KLG of no more than $5 \mathrm{~g} / \mathrm{L}$ [9].

G. oxydans is an industrially important bacterium for its ability to oxidize sugars and sugar alcohols at high levels. Many compounds such as acetic acid, D-gluconic acid, L-sorbose, and dihydroxyacetone have been produced successfully with G. oxydans [10-14]. In industrial 2-KLG synthesis, G. oxydans was initially found to be used in the Reichstein process for the oxidation of D-sorbitol to L-sorbose, which is a species of choice for the construction of genetically engineered strain that equipped the crucial dehydrogenases required for the conversion of D-sorbitol to 2KLG [15]. G. oxydans WSH-003, in this study, is an L-sorbose-accumulating strain of industrial interest due to its powerful ability to oxidize D-sorbitol into L-sorbose with a high quantitative yield of over $98 \%$ on an industrial scale. G. oxydans WSH-003 was mutated by different methods from a wild-type strain for at least 90 times to improve the production of L-sorbose and the tolerance to saccharides and alditols. Finally, the industrial strain possessed both high L-sorbose productivity and extreme tolerance to saccharides and alditols [16]. In addition, in our previous study, it was identified that even $100 \mathrm{~g} / \mathrm{L}$ of 2 -KLG did not obviously affect the cell growth of G. oxydans WSH-003 and no obvious degradation of $2-\mathrm{KLG}$ could be detected when $G$. oxydans WSH-003 was grown with 2-KLG [9].

Following the development of next-generation sequencing technology, the three bacteria involved in the classical two-step fermentation process for L-AA production have all been sequenced in my original laboratory. The gene clusters encoding $\mathrm{D}$-sorbitol dehydrogenase and responsible for the synthesis of the cofactor pyrroloquinoline quinone (PQQ) were identified from the genome sequence of $G$. oxydans WSH-003 [16]. Meanwhile, in K. vulgare WSH-001, five L-sorbose dehydrogenases (SDHs) and two L-sorbosone dehydrogenases (SNDHs) were confirmed by expression of the DNA in Escherichia coli, which were a group of PQQdependent dehydrogenases for the catalysis of L-sorbose to L-sorbosone and further to 2-KLG [17]. In our previous study, five SDH genes and two SNDH genes were overexpressed in G. oxydans with different combinations, and by a series of biological engineering, the 2-KLG production increased to $32.4 \mathrm{~g} / \mathrm{L}$. In this study, the cross-linker protein CutA was used as a protein scaffold to improve the chemical structure stability of SDH and SNDH, which was the first time for the application of CutA in metabolic engineering. The recombinant strain pGUC-tufB-SH3-sdh-GGGGSsndh-tufB-SH3 $3_{\text {lig }}$ (GGGGS) $)_{2}$-cutA produced $40.3 \mathrm{~g} / \mathrm{L}$ of $2-$ KLG after $168 \mathrm{~h}$, and the production was improved efficiently. Furthermore, the expression levels of the cofactor PQQ were enhanced to further improve 2-KLG production. With the stepwise metabolic engineering of $G$. oxydans, the final 2-KLG production was improved to $42.6 \mathrm{~g} / \mathrm{L}$, which was $5.7 \%$ higher than that by pGUC-tufB-SH3-sdh-GGGGSsndh-tufB-SH3 $3_{\text {lig }}$ (GGGGS) $)_{2}$-cutA.

\section{Materials and Methods}

2.1. Strains and Plasmids. K. vulgare WSH-001 and G. oxydans WSH-003 were provided by Jiangsu Jiangshan Pharmaceutical Co., Ltd. Escherichia coli JM109 (Novagen, Darmstadt, Germany) was used as the host for plasmid construction. The pMD19-T vector was used for gene cloning (TakaRa, Dalian, China). All plasmids used in this study are provided in Table 1. PrimeSTAR HS DNA polymerase, restriction endonucleases, DNA gel extraction kit, and PCR reagents were purchased from TakaRa (Dalian, China). FastPure DNA kit and SanPrep Column Plasmid Mini-Preps Kit were purchased from Sangon (Shanghai, China). DNA Sanger sequencing was performed by Sangon (Shanghai, China).

2.2. Culture Conditions. G. oxydans strains were cultivated in a broth $(15 \%$ D-sorbitol, $1.5 \%$ corn steep liquor, and $2 \%$ $\mathrm{CaCO}_{3}$ ) at $30^{\circ} \mathrm{C}$ for $168 \mathrm{~h}$. G. oxydans transformants were cultivated in medium containing $75 \mu \mathrm{g} / \mathrm{mL}$ ampicillin. E. coli strains were cultivated in the Luria-Bertani (LB, Oxoid) medium. $100 \mu \mathrm{g} / \mathrm{mL}$ of ampicillin was added to the LB medium to screening transformants with plasmids.

2.3. Codon Optimization of SDH and SNDH. The codons of the $s d h$-sndh gene (the two genes were fused with GGGGS linker peptide) were optimized based on the codon preference of $G$. oxydans using a codon algorithm with the GeMS software [18]. The codon-optimized gene was synthetized by Genewiz (Nanjing, China).

2.4. Expression of Trimeric Protein CutA in G. oxydans WSH003. CutA is a small trimeric protein from Pyrococcus horikoshii (GenBank Accession number: BAA30089.1) [19]. In order to verify whether the expression of cutA could take effect in G. oxydans, cutA was optimized as stated above and was synthetized by Genewiz. The fragment of the codonoptimized cutA was digested and inserted into the KpnI/ BamHI site of pGUC (the shuttle vector of E. coli-G. oxydans that constructed in our previous study). Then, the promoter of elongation factor TU (tufB) [20] was inserted into the SacI/KpnI site of pGUC-cutA, resulting in pGUC-tufB-cutA, 
TABle 1: Plasmids used in this study.

\begin{tabular}{|c|c|c|}
\hline Plasmids & Relevant characteristics & Sources \\
\hline pGUC & Ampr E. coli-G. oxydans shuttle vector & This study \\
\hline pGUC-tufB-sdh-GGGGS-sndh & pGUC containing tufB-sdh-GGGGS-sndh & This study \\
\hline pGUC-tufB-cutA & pGUC containing tufB-cutA & This study \\
\hline $\begin{array}{l}\text { pGUC-tufB-SH3-sdh-GGGGS-sndh-tufB-SH3 } \text { lig- }^{-} \\
\text {(GGGGS) }{ }_{2} \text {-cutA }\end{array}$ & $\begin{array}{l}\text { pGUC containing tufB-SH3-sdh-GGGGS-sndh-tufB-SH3 } \text { lig- }^{-} \\
(\mathrm{GGGGS})_{2}-\text { cutA }\end{array}$ & This study \\
\hline $\begin{array}{l}\text { pGUC-tufB-SH3-sdh-GGGGS-sndh-tufB-SH3} \text { lig- }^{-} \\
\text {(GGGGS) })_{2} \text {-cutA-tufB-pqqABCDE }\end{array}$ & $\begin{array}{l}\text { pGUC containing tufB-SH3-sdh-GGGGS-sndh-tufB-SH3} 3_{\text {lig- }} \\
(\text { GGGGS })_{2}-c u t A-t u f B-p q q A B C D E\end{array}$ & This study \\
\hline
\end{tabular}

and was transformed into G. oxydans WSH-003 by electroporation [21].

2.5. Fusion Expression of SDH and SNDH with CutA. For the ligation of SDH-GGGGS-SNDH with CutA, adaptor protein $(\mathrm{SH} 3)$ and its ligand $\left(\mathrm{SH}_{3}\right.$ lig $)$ were used as the docking protein and docking station peptide, respectively [22-24]. Both SH3 and $\mathrm{SH}_{\text {lig }^{-}}(\mathrm{GGGGS})_{2}$ - (utA were optimized as stated above and were synthetized by Genewiz. The $\mathrm{SH} 3$ was fused with codon-optimized $s d h$-GGGGS-sndh, resulting in SH3-sdh-GGGGS-sndh. The $S H 3_{\text {lig- }}(\mathrm{GGGGS})_{2}$-cutA was fused with the promoter $t u f B$, resulting in tufB-SH $3_{\text {lig }^{-}}$ (GGGGS) $)_{2}-c u t A$. The obtained SH3-sdh-GGGGS-sndh and tufB-SH3 $3_{\text {lig }}$-(GGGGS $)_{2}$-cutA were further digested and inserted into the KpnI/BamHI and BamHI/XbaI site of pGUC-tufB, respectively, resulting in pGUC-tufB-SH3-sdh-

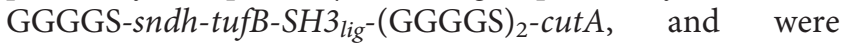
transformed into $G$. oxydans WSH-003 by electroporation [21]. The time courses of sorbitol oxidation by the recombinant strains were performed, every $12 \mathrm{~h}$ to take a sample, and were analyzed by HPLC. The mean value out of three independent experiments was calculated.

2.6. Overexpression of the Cofactor PQQ in G. oxydans WSH003. pqqABCDE was amplified using G. oxydans WSH-003 genomic DNA with the primers (Table 2). Because of the lack of restriction enzyme site, the promoter $t u f B$ was fused with pqqABCDE, which was digested and inserted into the $\mathrm{XbaI} /$ PstI site of pGUC, resulting in pGUC-tufB-SH3-sdh-GGGGSsndh-tufB-SH3 $3_{\text {lig }}$-(GGGGS $)_{2}$-cutA-pqqABCDE. The construct was transformed into G. oxydans WSH-003 by electroporation [21].

2.7. Analysis Procedures. The optical density of the culture broth was measured using a Biospec-1601 spectrophotometer (Shimadzu, Kyoto, Japan) at $600 \mathrm{~nm}$ after an appropriate dilution. D-sorbitol, 2-KLG, and intermediate metabolites in the fermentation broth were determined by HPLC, using an Aminex HPX-87H column (Bio-Rad, Richmond, CA) at $35^{\circ} \mathrm{C}$ with a flow rate of $0.6 \mathrm{~mL} / \mathrm{min}$ and $5 \mathrm{mmol} / \mathrm{L} \mathrm{H}_{2} \mathrm{SO}_{4}$ as the eluent [25-27]. The concentration of PQQ in the culture supernatants was measured using LC-MS-IT-TOF under the conditions reported by Noji et al. [28].

\section{Results and Discussion}

3.1. Overexpression of SDH and SNDH in G. oxydans WSH003 after Codon Optimization. G. oxydans WSH-003 is an industrial strain with high L-sorbose productivity and extreme tolerance to saccharides and alditols. The metabolic pathway of D-sorbitol in the recombinant G. oxydans strains is shown in Figure 1. In our previous study, five SDHs (KVU_pmdA_0245, KVU_2142, KVU_2159, KVU_1366, and KVU_0203) and two SNDHs (KVU_0095 and KVU_pmdB_0115) in K. vulgare WSH-001 were identified [17], which were introduced into G. oxydans WSH-003 in different combinations to construct the one-step strain. After a series of biological engineering, the production of 2KLG was up to $32.4 \mathrm{~g} / \mathrm{L}$ after $168 \mathrm{~h}$ of fermentation by $G$. oxydans/pGUC-tufB-k0203-GGGGS-k0095 (G. oxydans/ pGUC-tufB-sdh-GGGGS-sndh) [9].

Codon optimization is a key technique to achieve the efficient expression of heterologous proteins. Codon preference optimization strategy is the most commonly used codon optimization strategy at present, which mainly replaces the donor codon with the synonymous codon with the highest frequency in the host genome and uses the most abundant codon in the host to encode the amino acids in the optimized sequence [29]. In this study, codon preference optimization was conducted to further enhance the efficiency of expression of SDH and SNDH in G. oxydans. The $s d h$-GGGGS-sndh shares $82 \%$ similarity with its parental nucleotide sequence while maintaining the identical amino acid sequence. After $168 \mathrm{~h}$ of fermentation, the 2-KLG production reached $33.2 \mathrm{~g} / \mathrm{L}$. It was not significant for the enhancement of 2-KLG production by codon optimization, which may be due to the complex and varied factors, such as posttranslational folding and metabolic level, and thus, the expression of SDH and SNDH remains low [30].

3.2. Expression of Trimeric Protein CutA in G. oxydans WSH003. CutA is a small trimeric protein from P. horikoshii, which is used as the cross-linker protein [19]. It has an extremely high denaturation temperature of nearly $150^{\circ} \mathrm{C}$ [31]. Furthermore, CutA retains its trimeric quaternary structure in a solution containing as much as $5 \mathrm{M} \mathrm{GuHCl}$ [32]. It was reported that the remarkably increased number of ion pairs in the monomeric structure contributes to the stabilization of the trimeric structure and plays an important 
Table 2: Primers used in this study.

\begin{tabular}{|c|c|c|}
\hline Primer & Sequences of primers $\left(5^{\prime}-3^{\prime}\right)^{*}$ & Restriction enzyme \\
\hline tufB-F1 & CGAGCTCGTACGATGGTAAGAAATCCACTG & SacI \\
\hline tufB-R1 & CGGGGTACCCGTCTTTCTCCAAAACCCC & KpnI \\
\hline SH3-F & CGGGGTACCGCCGAGTATGTGCGCGCCCT & KpnI \\
\hline SH3-R & GGGTCGTGAGCTTCATGTACTTCTCCACGTACGGCACCG & - \\
\hline sdh-GGGGS-sndh-F & GTACGTGGAGAAGTACATGAAGCTCACGACCCTGCTGC & - \\
\hline sdh-GGGGS-sndh-R & CGCGGATCCTCACGCCGCGGAAATCCGC & BamHI \\
\hline tufB-F2 & CGCGGATCCGTACGATGGTAAGAAATCCACTG & BamHI \\
\hline tufB-R2 & GCAGGGCCGGCGGCGGCGTCTTTCTCCAAAACCCCGCT & - \\
\hline $\mathrm{SH}_{\text {lig }}-(\mathrm{GGGGS})_{2}$-cutA-F & AGCGGGGTTTTGGAGAAAGACGCCGCCGCCGGCCCTGC & - \\
\hline $\mathrm{SH}_{3}$ lig $_{-}^{\circ}(\mathrm{GGGGS})_{2}-\mathrm{cutA}-\mathrm{R}$ & CTAGTCTAGATCACTTCTTCGTCTCCTCGATCAGC & XbaI \\
\hline tufB-F3 & CTAGTCTAGATGCAGATCCGGTGGCCATGTTC & XbaI \\
\hline tufB-R3 & TTCGGCGTGTTCCAAGCCATCGTCTTTCTCCAAAACCCCGCT & - \\
\hline pqqABCDE-F & CGGGGTTTTGGAGAAAGACGATGGCTTGGAACACGCCG & - \\
\hline pqqABCDE-R & AAAACTGCAGTTACATTCTTCGGTAAACAAAGT & PstI \\
\hline
\end{tabular}

${ }^{*}$ Restriction sites used for cloning are in bold and are underlined.

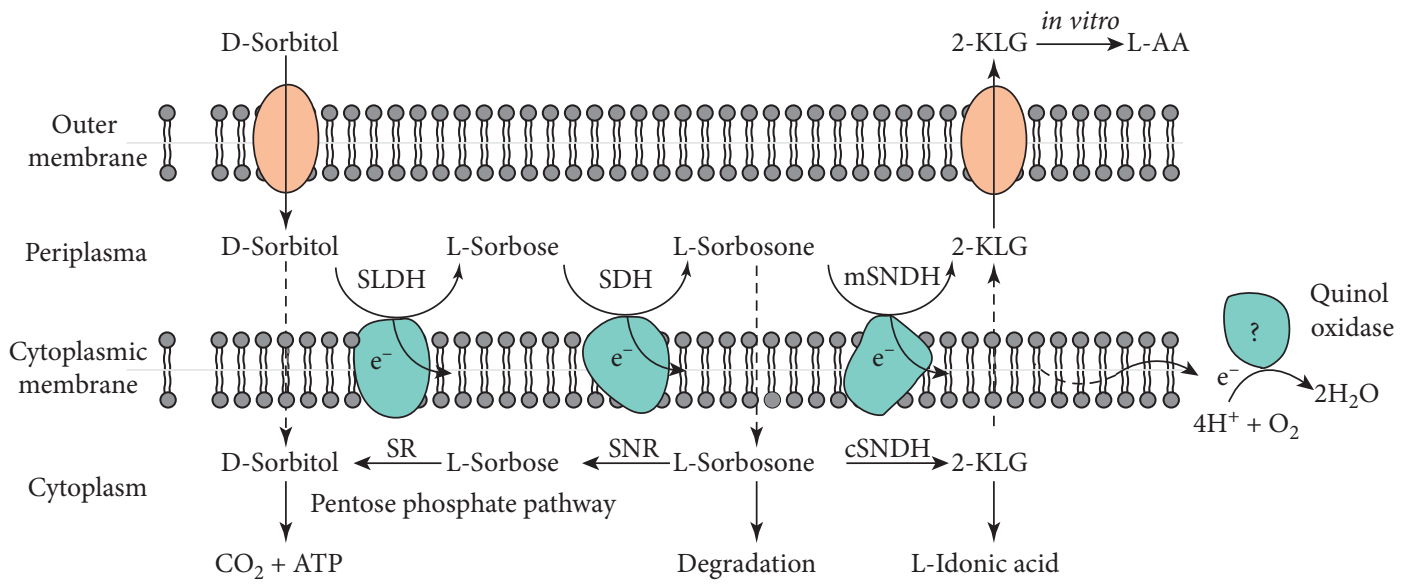

Figure 1: The metabolic pathway of D-sorbitol in the recombinant G. oxydans strains. SLDH, D-sorbitol dehydrogenase; SDH, L-sorbose dehydrogenase; SNDH, L-sorbosone dehydrogenase; mSNDH, membrane-bound SNDH; cSNDH, cytosolic SNDH; SR, L-sorbose reductase; SNR, L-sorbosone reductase.

role in enhancing the denaturation temperature up to $150^{\circ} \mathrm{C}$ [31].

Overexpression of CutA in G. oxydans WSH-003 was conducted to verify whether it would influence the growth of the strain. After the codon optimization of cutA, G. oxydans strain harboring pGUC-tufB-cutA was constructed. The growth curve of G. oxydans/pGUC-tufB-cutA and the wild strain was determined (Figure 2 ). The results showed that the growth was consistent with each other at $30^{\circ} \mathrm{C}$, and $G$. oxydans/pGUC-tufB-cutA grew faster at $37^{\circ} \mathrm{C}$ and $42^{\circ} \mathrm{C}$ than the wild strain. After $12 \mathrm{~h}$ of fermentation, the $\mathrm{OD}_{600}$ of $G$. oxydans/pGUC-tufB-cut $A$ and the wild strain at $37^{\circ} \mathrm{C}$ was 6.74 and 5.99, respectively, and at $42^{\circ} \mathrm{C}$ was 5.31 and 4.1 , respectively. However, the growth of $G$. oxydans/pGUCtufB-cutA and the wild strain was both inhibited at $45^{\circ} \mathrm{C}$. It was found that the expression of the cross-linker protein CutA improved the heat resistance of the strain.

3.3. Overexpression of SDH and SNDH in G. oxydans WSH003 Based on CutA. Ramirez et al. developed a novel self- assembling protein hydrogel with cross-linked protein CutA and formed a highly cross-linked protein network. The building blocks initiate an intein trans-splicing reaction that yields a hydrogel that is highly stable over a wide range of $\mathrm{pH}$ (6-10) and temperature $\left(4-50^{\circ} \mathrm{C}\right)$ [22]. Inspired by this study, the stability of SDH and SNDH may be improved by generating a longer protein chain with the cross-linker CutA, and the catalytic efficiency of the enzymes may be increased further.

The trimeric protein CutA from P. horikoshii was able to be used as the protein scaffold, which may improve the stability of SDH and SNDH in G. oxydans. The adaptor protein $\mathrm{SH} 3$ and its ligand $\mathrm{SH}_{3}$ lig were used as docking protein and docking station peptide, respectively. They possess high affinity for each other because of their relatively small size (56 and 11 amino acids, respectively) (Figure 3). After $\mathrm{SH} 3$ and $\mathrm{SH}_{\text {lig }}$-(GGGGS) ${ }_{2}$-cutA were optimized and synthetized, they were fused or ligated with the promoter tufB and codon-optimized sdh-GGGGS-sndh, and a recombinant plasmid pGUC-tufB-SH3-sdh-GGGGS-sndhtufB-SH3 ${ }_{\text {lig }}(\mathrm{GGGGS})_{2}$-cutA was constructed and was 


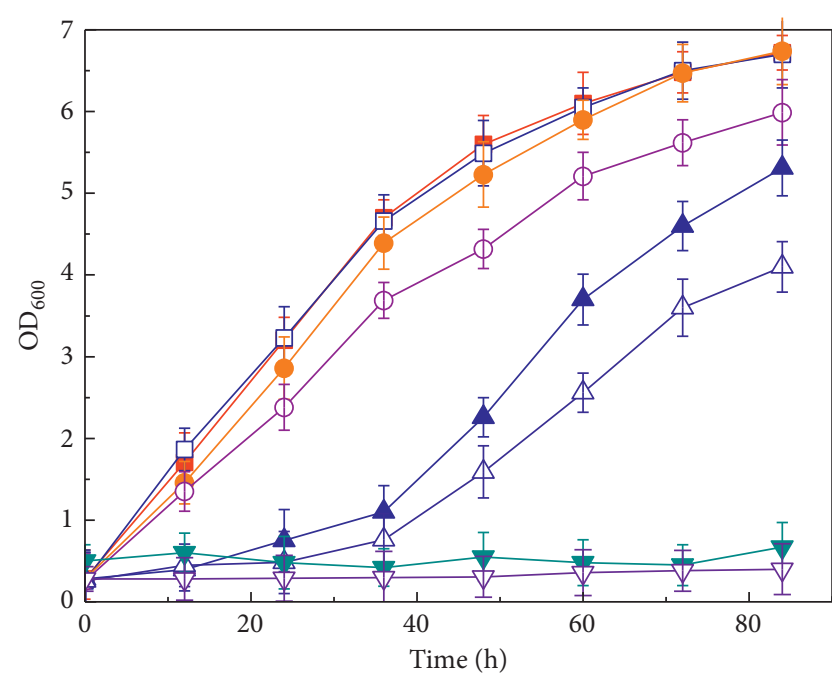

FIgURE 2: The effect on temperature tolerance of G. oxydans after the expression of CutA. The symbols $\boldsymbol{\square}, \boldsymbol{\bullet}, \boldsymbol{\Delta}$, and $\boldsymbol{\nabla}$ represent the growth curve of G. oxydans/pGUC-tufB-cutA at $30^{\circ} \mathrm{C}, 37^{\circ} \mathrm{C}, 42^{\circ} \mathrm{C}$, and $45^{\circ} \mathrm{C}$, respectively. The symbols $\square, \bigcirc, \triangle$, and $\nabla$ represent the growth curve of $\mathrm{G}$. oxydans $/ \mathrm{pGUC}$ at $30^{\circ} \mathrm{C}, 37^{\circ} \mathrm{C}, 42^{\circ} \mathrm{C}$, and $45^{\circ} \mathrm{C}$, respectively. Error bars represent the standard deviation of three biological replicates.

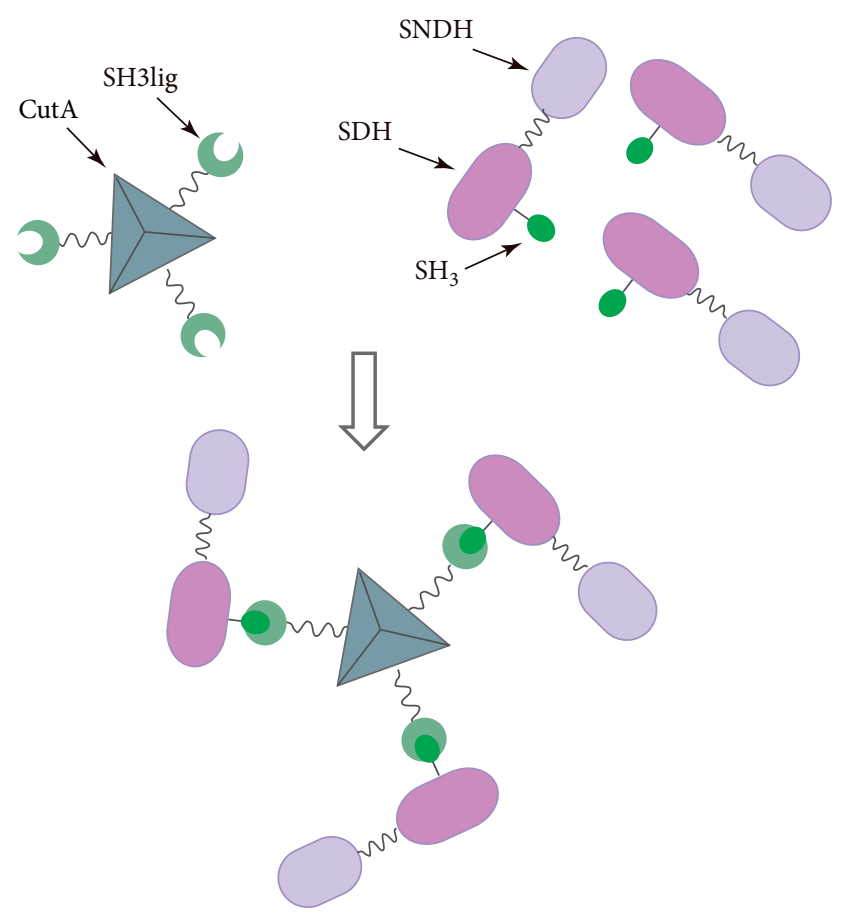

FIgURE 3: The scheme of protein expression based on the trimeric protein CutA. A longer protein chain with the trimeric CutA was generated under the use of the adaptor protein $\mathrm{SH} 3$ and its ligand $\mathrm{SH}_{\text {lig }}$ as docking protein and docking station peptide, respectively.

transferred into G. oxydans WSH-003. Comparison of optical densities at $600 \mathrm{~nm}\left(\mathrm{OD}_{600}\right)$ and product formation in the recombinant strains is shown in Figure 4. G. oxydans/ pGUC-tufB-SH3-sdh-GGGGS-sndh-tufB-SH3 lig (GGGGS) ${ }_{2}$ cutA grew basically consistent with G. oxydans/pGUC-tufB- $s d h$-GGGGS-sndh, and the 2-KLG production by the G. oxydans strain harboring pGUC-tufB-SH3-sdh-GGGGSsndh-tufB-SH3 ${ }_{\text {lig }}$ (GGGGS) ${ }_{2}$-cutA was $40.3 \mathrm{~g} / \mathrm{L}$ after $168 \mathrm{~h}$ of fermentation, which was $24.4 \%$ higher than that obtained by G. oxydans/pGUC-tufB-sdh-GGGGS-sndh, indicating that application of the cross-linker protein CutA efficiently increased the yield of 2-KLG.

3.4. Fermentation of Engineering G. oxydans at Different Temperatures. In the above study, it has been proved that the expression of CutA makes the strain more tolerable to temperature. Therefore, in order to investigate the fermentation performance at different temperatures, the recombinant strain G. oxydans/pGUC-tufB-SH3-sdhGGGGS-sndh-tufB-SH3 $3_{\text {lig }}$-(GGGGS) ${ }_{2}$-cutA was fermented at $30^{\circ} \mathrm{C}, 35^{\circ} \mathrm{C}$, and $37^{\circ} \mathrm{C}$, respectively. The recombinant strain G. oxydans/pGUC-tufB-sdh-GGGGS-sndh was used as control. The $\mathrm{OD}_{600}$ and the production of $2-\mathrm{KLG}$ at different temperatures are summarized in Figure 5. The results showed that the growth of G. oxydans/pGUC-tufB-SH3-sdhGGGGS-sndh-tufB-SH3 ${ }_{\text {lig }}$-(GGGGS) ${ }_{2}$-cutA was nearly consistent with each other at these three temperatures, and it grew better at $35^{\circ} \mathrm{C}$ and $37^{\circ} \mathrm{C}$ than the control strain. The production of 2-KLG of G. oxydans/pGUC-tufB-SH3-sdhGGGGS-sndh-tufB-SH3 ${ }_{\text {lig }}$-(GGGGS) ${ }_{2}$-cutA was higher than the control at different temperatures. Furthermore, the specific activity of SDH and SNDH in the recombinant and the control strain at different temperatures is summarized in Figure 6. The results showed that the specific activity of SDH and SNDH was also higher than that of the control at different temperatures, which revealed that the stability and catalytic efficiency of the dehydrogenases may be improved after the expression of CutA. However, both strains of $G$. oxydans/pGUC-tufB-SH3-sdh-GGGGS-sndh-tufB-SH3 ${ }_{\text {lig- }}$ (GGGGS) ${ }_{2}$-cutA and the control produced less $2-\mathrm{KLG}$ at $35^{\circ} \mathrm{C}$ and $37^{\circ} \mathrm{C}$ than at $30^{\circ} \mathrm{C}$. This may be caused by that the enzyme activities were affected at higher temperatures.

3.5. Overexpression of pqqABCDE to Improve the 2-KLG Production. It has been confirmed that G. oxydans WSH003 possesses a complete PQQ synthesis and regeneration system. However, the biosynthesis of 2-KLG is an oxidative process, and a large amount of the reduced cofactors, such as $\mathrm{PQQH}_{2}$, should be rapidly regenerated for the following oxidation process. This means that the introduction of the extra PQQ-dependent dehydrogenases could lead to cofactor imbalances in metabolic pathways and significantly increase the burden for the global metabolic networks, thereby affecting 2-KLG production [33]. Cofactor engineering is often adopted to compensate the imbalance of cofactors to improve product synthesis [34]. In our previous study, SDH and SNDH from K. vulgare WSH-001 and SLDH from G. oxydans WSH-003 were all identified as PQQ-dependent dehydrogenases, and it was noted that increasing PQQ level could further improve the production of 2-KLG [9]. In this study, pGUC-tufB-SH3-sdh-GGGGS-sndh-tufB$\mathrm{SH}_{\text {lig }}$-(GGGGS) $)_{2}$-cutA-tufB-pqqABCDE was constructed. The production of 2-KLG by G. oxydans strain harboring 


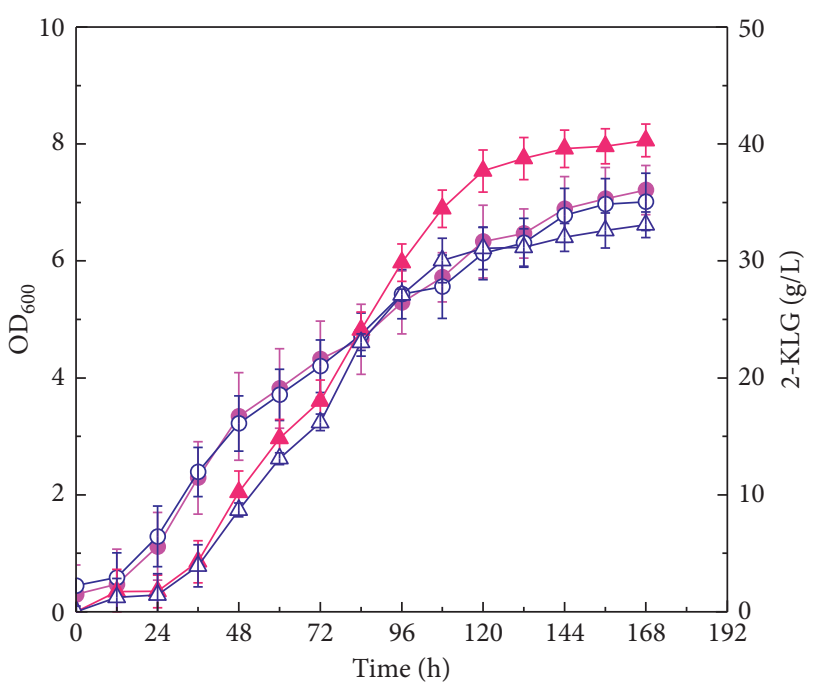

FIgURE 4: Time course of fermentation by CutA-based engineered strain. The symbols $\boldsymbol{\Delta}$ and $\triangle$ represent the concentration of 2KLG produced by $G$. oxydans/pGUC-tufB-SH3-sdh-GGGGSsndh-tufB-SH3 $3_{\text {lig }}$ (GGGGS) ${ }_{2}$-cutA and G. oxydans/pGUC-tufB$s d h$-GGGGS-sndh, respectively. The symbols $\bullet$ and $\bigcirc$ represent $\mathrm{OD}_{600}$ of G. oxydans/pGUC-tufB-SH3-sdh-GGGGS-sndh-tufB$S H 3_{\text {lig }}$ (GGGGS $)_{2}$-cutA and G. oxydans/pGUC-tufB-sdhGGGGS-sndh, respectively. Error bars represent the standard deviation of three biological replicates.

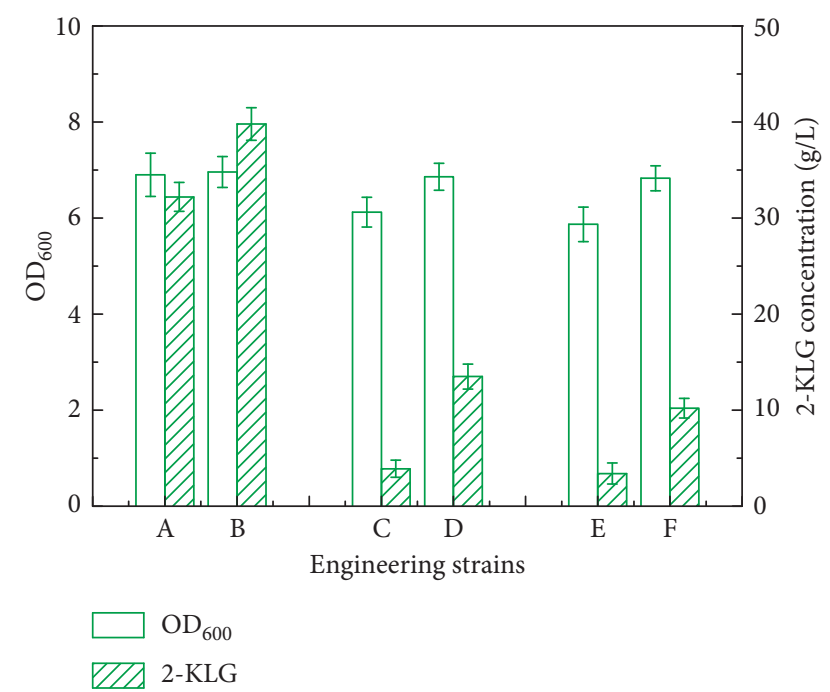

Figure 5: Production of 2-KLG by CutA-based engineered strain and the control strain at different temperatures. A, C, and E are $\mathrm{OD}_{600}$ and 2-KLG production of G. oxydans/pGUC-tufB-sdhGGGGS-sndh at $30^{\circ} \mathrm{C}, 35^{\circ} \mathrm{C}$, and $37^{\circ} \mathrm{C}$, respectively. $\mathrm{B}, \mathrm{D}$, and $\mathrm{F}$ are $\mathrm{OD}_{600}$ and 2-KLG production of G. oxydans/pGUC-tufB-SH3-sdhGGGGS-sndh-tufB-SH3 ${ }_{\text {lig }}-(\mathrm{GGGGS})_{2}$-cutA at $30^{\circ} \mathrm{C}, 35^{\circ} \mathrm{C}$, and $37^{\circ} \mathrm{C}$, respectively. Error bars represent the standard deviation of three biological replicates.

pGUC-tufB-SH3-sdh-GGGGS-sndh-tufB-SH3 ${ }_{\text {lig }}$ (GGGGS) 2- $_{2}$ cutA-tufB-pqqABCDE after $168 \mathrm{~h}$ of fermentation is summarized in Figure 7. Overexpression of pqqABCDE gene clusters enhanced PQQ production by $262.5 \%$ compared with

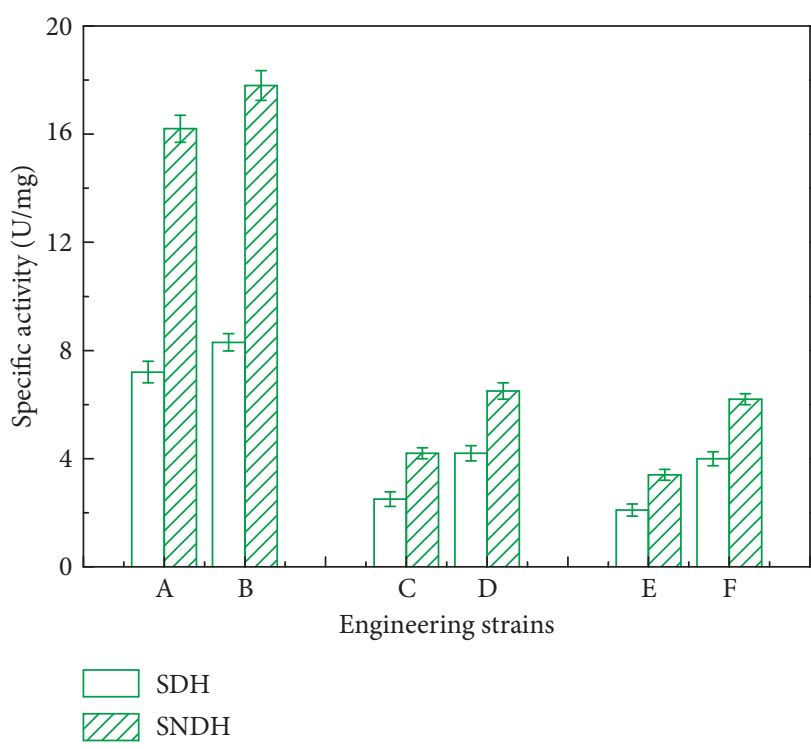

FIgURE 6: The specific activities of SDH and SNDH in the CutA-based engineered strain and the control strain at different temperatures. A, C, and $\mathrm{E}$ are SDH and SNDH specific activities of G. oxydans/pGUCtufB-sdh-GGGGS-sndh at $30^{\circ} \mathrm{C}, 35^{\circ} \mathrm{C}$, and $37^{\circ} \mathrm{C}$, respectively. $\mathrm{B}, \mathrm{D}$, and $\mathrm{F}$ are SDH and SNDH specific activities of $G$. oxydans/pGUC-tufBSH3-sdh-GGGGS-sndh-tufB-SH3 $3_{\text {lig- }}$-(GGGGS) ${ }_{2}$-cutA at $30^{\circ} \mathrm{C}, 35^{\circ} \mathrm{C}$, and $37^{\circ} \mathrm{C}$, respectively. Error bars represent the standard deviation of three biological replicates.

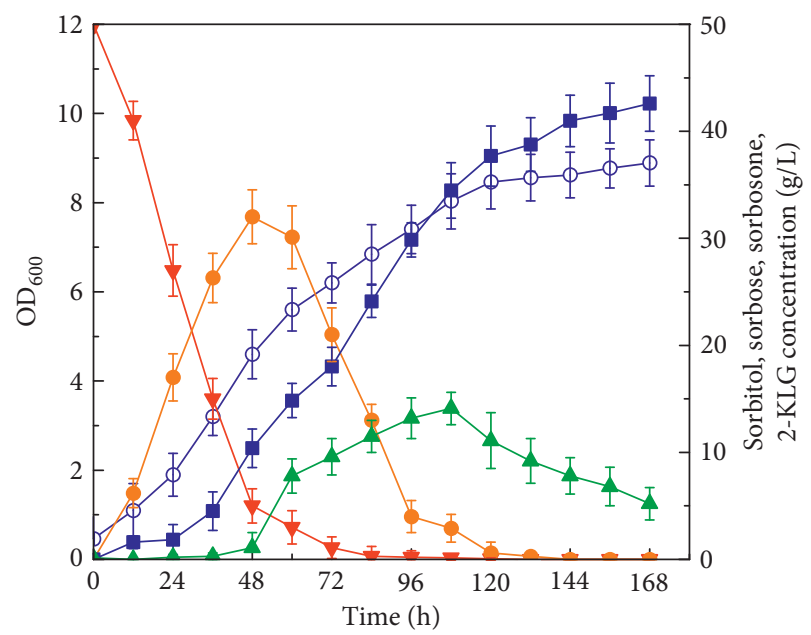

FIgURE 7: Time course of oxidative fermentation of D-sorbitol by the PQQ biosynthesis enhanced engineered strains. The symbols $\boldsymbol{\nabla}, \boldsymbol{\bullet}, \boldsymbol{\Delta}$, and represent the concentration of D-sorbitol, L-sorbose, L-sorbosone, and 2-KLG produced by G. oxydans/pGUC-tufB-SH3-sdhGGGGS-sndh-tufB-SH3 $3_{\text {lig }}$ (GGGGS) 2 $_{2}$-cutA-tufB-pqqABCDE, respectively. The symbol $O$ represents $\mathrm{OD}_{600}$ of $G$. oxydans/pGUC-tufB-SH3$s d h$-GGGGS-sndh-tufB-SH3 ${ }_{\text {lig- }}$ (GGGGS) $)_{2}$-cutA-tufB-pqqABCDE, respectively. Error bars represent the standard deviation of three biological replicates.

the wild-type strain. Furthermore, overexpression of PQQ biosynthesis genes significantly enhanced cell growth, and the likely reason for this is that PQQ is also a signaling molecule in signal transduction pathways that affect bacterial growth by 
neutralizing reactive species $[35,36]$. The $2-\mathrm{KLG}$ production by $G$. oxydans/pGUC-tufB-SH3-sdh-GGGGS-sndh-tufB$S H 3_{\text {lig }}$ (GGGGS) ${ }_{2}$-cutA-tufB-pqqABCDE reached $42.6 \mathrm{~g} / \mathrm{L}$, which was $5.7 \%$ higher than that by G. oxydans/pGUC-tufBSH3-sdh-GGGGS-sndh-tufB-SH3 lig $_{\text {- }}$ (GGGGS) $)_{2}$-cutA. Unfortunately, the overexpression of $p q q \mathrm{ABCDE}$ did not increase the yield significantly, but the fermentation cycle could be shortened.

In the past several decades, tremendous advances have been achieved in VC production by the classical two-step fermentation process. However, the further decreasing of $\mathrm{VC}$ price is significantly restricted because of many problems such as the two times of sterilization and mix-culture fermentation. Therefore, much more attention has been focused on the development of one-step fermentation route, but it is to be regretted that the progress is not ideal. In this study, the production of $2-\mathrm{KLG}$ reached $42.6 \mathrm{~g} / \mathrm{L}$ with the stepwise metabolic engineering of $G$. oxydans. Nowadays, with the development of biological technology, many new metabolic engineering methods were developed. The comprehensive optimization of metabolic engineering strategies and fermentation optimization should further facilitate the research process for VC production by one-step fermentation route.

\section{Conclusions}

In summary, SDH and SNDH were expressed in G. oxydans after the codon optimization. Furthermore, the trimeric protein CutA was used to improve the chemical structure stability of SDH and SNDH. The recombinant strain $G$. oxydans/pGUC-tufB-SH3-sdh-GGGGS-sndh-tufB-SH3 lig- $^{-}$ (GGGGS) $)_{2}$-cutA produced $40.3 \mathrm{~g} / \mathrm{L}$ of $2-\mathrm{KLG}$ after $168 \mathrm{~h}$. In addition, the expression levels of the cofactor PQQ were enhanced to further improve 2-KLG production. With the stepwise metabolic engineering of G. oxydans, the final 2-KLG production was improved to $42.6 \mathrm{~g} / \mathrm{L}$. Efficient one-step production of $2-$ KLG was achieved.

\section{Data Availability}

The data used to support the findings of this study are included within the article.

\section{Conflicts of Interest}

The authors declare that there are no conflicts of interest regarding the publication of this paper.

\section{Acknowledgments}

This work was supported by grants from the Natural Science Foundation of Hebei Province (no. C2018203374).

\section{References}

[1] P. Wang, W. Zeng, S. Xu, G. Du, J. Zhou, and J. Chen, "Current challenges facing one-step production of L-ascorbic acid," Biotechnology Advances, vol. 36, no. 7, pp. 1882-1899, 2018.
[2] Mandlaa, Z. Sun, R. Wang et al., "Enhanced 2-keto-L-gulonic acid production by applying L-sorbose-tolerant helper strain in the co-culture system," AMB Express, vol. 8, pp. 1-7, 2018.

[3] Y. Zou, M. Hu, Y. Lv et al., "Enhancement of 2-keto-gulonic acid yield by serial subcultivation of co-cultures of Bacillus cereus and Ketogulonigenium vulgare," Bioresource Technology, vol. 132, pp. 370-373, 2012.

[4] C.-H. Pan, E.-X. Wang, N. Jia et al., "Reconstruction of amino acid biosynthetic pathways increases the productivity of 2keto-L-gulonic acid in Ketogulonicigenium vulgare-Bacillus endophyticus consortium via genes screening," Journal of Industrial Microbiology \& Biotechnology, vol. 44, no. 7, pp. 1031-1040, 2017.

[5] C. Wang, Y. Li, Z. Gao et al., "Establishing an innovative carbohydrate metabolic pathway for efcient production of 2keto-L-gulonic acid in Ketogulonicigenium robustum initiated by intronic promoters," Microbial Cell Factories, vol. 17, pp. 81-94, 2018.

[6] Y. Zhu, J. Liu, G. Du, J. Zhou, and J. Chen, "Sporulation and spore stability of Bacillus megaterium enhance Ketogulonigenium vulgare propagation and 2-keto-L-gulonic acid biosynthesis," Bioresource Technology, vol. 107, pp. 399-404, 2012.

[7] R. D. Hancock and R. Viola, "Biotechnological approaches for L-ascorbic acid production," Trends in Biotechnology, vol. 20, no. 7, pp. 299-305, 2002.

[8] Y. Saito, Y. Ishii, H. Hayashi et al., "Cloning of genes coding for L-sorbose and L-sorbosone dehydrogenases from Gluconobacter oxydans and microbial production of 2-keto-L-gulonate, a precursor of L-ascorbic acid, in a recombinant G. oxydans strain," Applied Environmental Microbiology, vol. 63, no. 2, pp. 454-460, 1997.

[9] L. Gao, Y. Hu, J. Liu, G. Du, J. Zhou, and J. Chen, "Stepwise metabolic engineering of Gluconobacter oxydans WSH-003 for the direct production of 2-keto-L-gulonic acid from D-sorbitol," Metabolic Engineering, vol. 24, pp. 30-37, 2014.

[10] M.-H. Li, J. Wu, X. Liu, J.-P. Lin, D.-Z. Wei, and H. Chen, "Enhanced production of dihydroxyacetone from glycerol by overexpression of glycerol dehydrogenase in an alcohol dehydrogenase-deficient mutant of Gluconobacter oxydans," Bioresource Technology, vol. 101, no. 21, pp. 8294-8299, 2010.

[11] X. Lin, S. Liu, G. Xie, J. Chen, P. Li, and J. Chen, "Enhancement of 1,3-dihydroxyacetone production from Gluconobacter oxydans by combined mutagenesis," Journal of Microbiology and Biotechnology, vol. 26, no. 11, pp. 19081917, 2016.

[12] X. Hua, G. Du, and Y. Xu, "Cost-practical of glycolic acid bioproduction by immobilized whole-cell catalysis accompanied with compressed oxygen supplied to enhance mass transfer," Bioresource Technology, vol. 283, pp. 326-331, 2019.

[13] X. Ke, Y. Pan-Hong, Z.-C. Hu, L. Chen, X.-Q. Sun, and Y.-G. Zheng, "Synergistic improvement of PQQ-dependent D-sorbitol dehydrogenase activity from Gluconobacter oxydans for the biosynthesis of miglitol precursor 6- $(\mathrm{N}$ hydroxyethyl)-amino-6-deoxy- $\alpha$-L-sorbofuranose," Journal of Biotechnology, vol. 300, pp. 55-62, 2019.

[14] S. Morena, M. G. Acedos, V. E. Santos, and F. García-Ochoa, "Dihydroxyacetone production from glycerol using Gluconobacter oxydans: study of medium composition and operational conditions in shaken flasks," Biotechnology Progress, vol. 35, pp. 2803-2811, 2019.

[15] U. Deppenmeier, M. Hoffmeister, and C. Prust, "Biochemistry and biotechnological applications of Gluconobacter strains," 
Applied Microbiology and Biotechnology, vol. 60, no. 3, pp. 233-242, 2002.

[16] L. Gao, J. Zhou, J. Liu, G. Du, and J. Chen, "Draft genome sequence of Gluconobacter oxydans WSH-003, a strain that is extremely tolerant of saccharides and alditols," Journal of Bacteriology, vol. 194, no. 16, pp. 4455-4456, 2012.

[17] L. Gao, G. Du, J. Zhou, J. Chen, and J. Liu, "Characterization of a group of pyrroloquinoline quinone-dependent dehydrogenases that are involved in the conversion of L-sorbose to 2-keto-L-gulonic acid in Ketogulonicigenium vulgare WSH001," Biotechnology Progress, vol. 29, no. 6, pp. 1398-1404, 2013.

[18] S. Jayaraj, R. Reid, and D. V. Santi, "GeMS: an advanced software package for designing synthetic genes," Nucleic Acids Research, vol. 33, no. 9, pp. 3011-3016, 2005.

[19] M. Sawano, H. Yamamoto, K. Ogasahara et al., "Thermodynamic basis for the stabilities of three CutA1s from Pyrococcus horikoshii, thermus thermophilus, and Oryza sativa, with unusually high denaturation temperatures," Biochemistry, vol. 47, no. 2, pp. 721-730, 2008.

[20] Y. Hu, H. Wan, J. Li, and J. Zhou, "Enhanced production of L-sorbose in an industrial Gluconobacter oxydans strain by identification of a strong promoter based on proteomics analysis," Journal of Industrial Microbiology \& Biotechnology, vol. 42, no. 7, pp. 1039-1047, 2015.

[21] L. Zhang, J. Lin, Y. Ma, D. Wei, and M. Sun, "Construction of a novel shuttle vector for use in Gluconobacter oxydans," Molecular Biotechnology, vol. 46, no. 3, pp. 227-233, 2010.

[22] M. Ramirez, D. Guan, V. Ugaz, and Z. Chen, "Intein-triggered artificial protein hydrogels that support the immobilization of bioactive proteins," Journal of the American Chemical Society, vol. 135 , no. 14 , pp. 5290-5293, 2013.

[23] L. Ge, M. Zhang, R. Wang et al., "Fabrication of CS/GA/RGO/ $\mathrm{Pd}$ composite hydrogels for highly efficient catalytic reduction of organic pollutants," RSC Advances, vol. 10, no. 26, pp. 15091-15097, 2020.

[24] Y. Feng, J. Yin, S. Liu, Y. Wang, B. Li, and T. Jiao, "Facile synthesis of Ag/Pd nanoparticle-loaded poly(ethylene imine) composite hydrogels with highly efficient catalytic reduction of 4-nitrophenol," ACS Omega, vol. 5, no. 7, pp. 3725-3733, 2020.

[25] J. Zhang, J. Liu, Z. Shi, L. Liu, and J. Chen, "Manipulation of $B$. megaterium growth for efficient 2-KLG production by $K$. vulgare," Process Biochemistry, vol. 45, no. 4, pp. 602-606, 2010.

[26] R. Wang, X. Yan, B. Ge et al., "Facile preparation of selfassembled black phosphorus-dye composite films for chemical gas sensors and surface-enhanced Raman scattering performances," ACS Sustainable Chemistry \& Engineering, vol. 8, no. 11, pp. 4521-4536, 2020.

[27] L. Zhang, J. Yin, K. Wei et al., "Fabrication of hierarchical SrTiO3@MoS2 heterostructure nanofibers as efficient and low-cost electrocatalysts for hydrogen-evolution reactions," Nanotechnology, vol. 31, no. 20, pp. 205604-205615, 2020.

[28] N. Noji, T. Nakamura, N. Kitahata et al., "Simple and sensitive method for pyrroloquinoline quinone (PQQ) analysis in various foods using liquid chromatography/electrosprayionization tandem mass spectrometry," Journal of Agricultural and Food Chemistry, vol. 55, no. 18, pp. 7258-7263, 2007.

[29] S. Tan, Y. Chen, Y. Gao et al., " $\beta$-Galactosidase gene codon optimization results in post-transcriptional enhancement of expression," Gene, vol. 748, 2020.
[30] T. E. F. Quax, N. J. Claassens, D. Söll, and J. van der Oost, "Codon bias as a means to fine-tune gene expression," Molecular Cell, vol. 59, no. 2, pp. 149-161, 2015.

[31] T. Tanaka, M. Sawano, K. Ogasahara et al., "Hyper-thermostability of CutA1 protein, with a denaturation temperature of nearly $150^{\circ} \mathrm{C}$," FEBS Letters, vol. 580, no. 17, pp. 4224-4230, 2006.

[32] Y. Tanaka, K. Tsumoto, T. Nakanishi et al., "Structural implications for heavy metal-induced reversible assembly and aggregation of a protein: the case of Pyrococcus horikoshii CutA1," FEBS Letters, vol. 556, no. 1-3, pp. 167-174, 2004.

[33] A. M. Sánchez, G. N. Bennett, and K.-Y. San, "Effect of different levels of NADH availability on metabolic fluxes of Escherichia coli chemostat cultures in defined medium," Journal of Biotechnology, vol. 117, no. 4, pp. 395-405, 2005.

[34] Y. Wang, K.-Y. San, and G. N. Bennett, "Cofactor engineering for advancing chemical biotechnology," Current Opinion in Biotechnology, vol. 24, no. 6, pp. 994-999, 2013.

[35] O. Choi, J. Kim, J.-G. Kim et al., "Pyrroloquinoline quinone is a plant growth promotion factor produced by Pseudomonas fluorescens B16," Plant Physiology, vol. 146, no. 2, pp. 657-668, 2008.

[36] M. Shrivastava, Y. S. Rajpurohit, H. S. Misra, and S. F. D'Souza, "Survival of phosphate-solubilizing bacteria against DNA damaging agents," Canadian Journal of Microbiology, vol. 56, no. 10, pp. 822-830, 2010. 